\title{
Up-regulation of NKG2F receptor, a functionally unknown killer receptor, of human natural killer cells by interleukin-2 and interleukin-15
}

\author{
HAIHUA HUANG，XIN WANG，YUKUN ZHANG，XIAODONG ZHENG，HAIMING WEI and RUI SUN
}

Institute of Immunology, Hefei National Laboratory for Physical Sciences at Microscale and School of Life Sciences, University of Science and Technology of China, Hefei, P.R. China

Received March 3, 2010; Accepted May 27, 2010

DOI: $10.3892 /$ or_00000953

\begin{abstract}
The NKG2 family receptors are C-type lectin, type II transmembrane molecules, and play important roles in regulation of natural killer (NK) cell functions against tumor and virus. NKG2F is a new member of NKG2 family, and may possibly associate with DAP 12 to activate NK cells. Since lacking available antibody against human $\mathrm{NKG} 2 \mathrm{~F}$, the features of NKG2F expression on NK cells remains unclear. In this study, human NKG2F recombinant expression in $E$. coli was carried out by using pET-28a with a hexahistidine (6X His) tag and a thrombin digestion sequence to the $\mathrm{N}$-terminus of the recombinant protein NKG2F. IPTG (isopropyl- $\beta$-d-thiogalactoside) induction resulted in high expression of recombinant $\mathrm{NKG} 2 \mathrm{~F}$ protein, which was then purified and identified by anti-His Western blotting and LC-MS/MS. Polyclonal antibody was produced by immunization of $\mathrm{BALB} / \mathrm{c}$ mice with recombinant $\mathrm{NKG} 2 \mathrm{~F}$, and then used to detect NKG2F in Western blotting and flow cytometry. Our results demonstrated that NKG2F was expressed only by PBMCs but not by human NK cell lines such as NKL and YT at mRNA level. It was observed that NKG2F was expressed on surface of human blood NK cells, and may be up-regulated at mRNA level and protein level after IL-2 or IL-15 stimulation.
\end{abstract}

\section{Introduction}

Natural killer (NK) cells play important roles in innate immunity. They are able to pan-specifically kill malignant cells or pathogen-infected cells without prior sensitization $(1,2)$. Previous study revealed that NK cell activation was balanced by inhibitory signals and activating signals through receptors on NK cell surface (3). Receptors of natural killer

Correspondence to: Dr Rui Sun, Institute of Immunology, School of Life Sciences, University of Science and Technology of China, 443 Huangshan Road, Hefei, Anhui 230027, P.R. China E-mail:sunr@ustc.edu.cn

Key words: recombinant expression, NK receptor, NKG2F, polyclonal antibody group 2 (NKG2) family are most important NK cell receptors with common protein structure of type II transmembrane and C-type lectin-like domain. The NKG2 receptors including NKG2-A, -B, -C, -D, -E, -F, and -H are membrane bound receptors, and usually form disulfide bonded heterodimers with the invariant CD94 chain (4). NKG2 receptors are divided into activating receptors and inhibitory receptors, depending on structural characteristics in their transmembrane or cytoplasmic tails $(5,6)$. The presence of a positively charged residue in the transmembrane region indicates the ability to associate with an activation mediating adaptor molecule (7), whereas the presence of an immunoreceptor tyrosine-based inhibition motif (ITIM) in the cytoplasmic tail suggests it as an inhibitory receptor (8). NKG2A/B has been shown to inhibit NK cell cytolytic activity through SHP-1 or SHP-2 recruitment by the phosphorylated ITIMs (9-11). NKG2C and NKG2E lack ITIMs in their cytoplasmic tails and associate with an immunoreceptor tyrosine-based activation motif (ITAM) bearing adaptor molecule, DAP12, through a positively charged lysine residue in the transmembrane region (12), thus they are considered as activating receptors. Cell surface expression of CD94/NKG2C is greatly enhanced by the association with DAP12, and the interaction of DAP12 and NKG2C depends on the charged residues in the transmembrane domains (12-14).

$\mathrm{NKG} 2 \mathrm{~F}$ is a new member of the NKG2 family receptors whose transcript has been detected. NKG2F gene has been identified to localize $25 \mathrm{~kb}$ from NKG2A as well as its relationship with the NKG2D c-DNA $(15,16)$. The putative human $\mathrm{NKG} 2 \mathrm{~F}$ protein is similar to $\mathrm{NKG} 2 \mathrm{C}$ with a lysine residue in the transmembrane region, but it has an ITIM-like motif in the cytoplasmic tail. It also has a relatively short extracellular region which does not contain a C-type lectin domain. Previous study has demonstrated that NKG2F is intrinsically expressed and may associate with DAP12 but not CD94. Moreover, the ITIM-like motif in its cytoplamic tail appears to be non-functional and NKG2F was considered as an activating receptor (17). However, the functions of NKG2F receptor have not been clarified yet. In this study, polyclonal antibody against recombinant human NKG2F was produced, and the NKG2F expression was observed to be up-regulated by IL-2 and IL-15 stimulation. Our results demonstrate that NKG2F might be possibly expressed on surface of human peripheral NK cells. 
A.

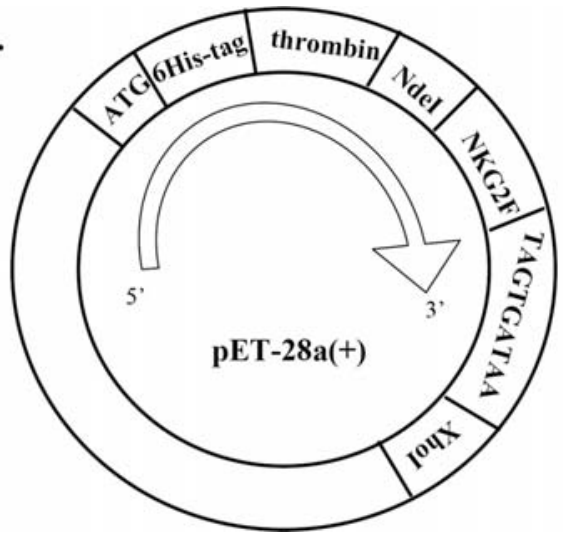

B.
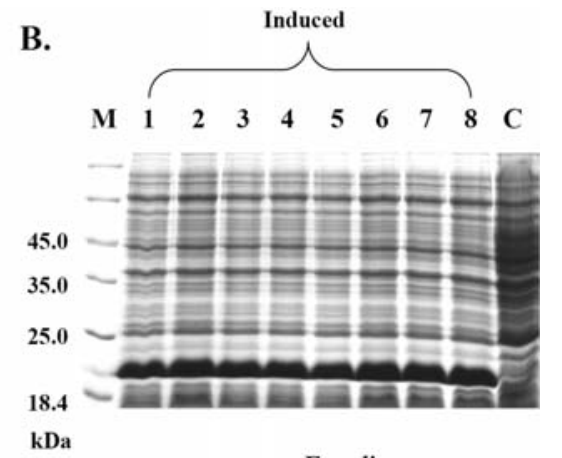

E. coli

Figure 1. Recombinant expression of human NKG2F. (A) Construction map of the recombinant NKG2F plasmid based on the commercial vector pET-28a (+). (B) SDS-PAGE analysis of E. coli protein samples. Lane M was the marker. Lanes 1-8 were eight $E$. coli clones induced by $0.1 \mathrm{mM}$ IPTG. Lane C was E. coli clone 8 without IPTG induction which was used as control. Dark lane at about $20 \mathrm{kDa}$ represents the recombinant NKG2F.

\section{Materials and methods}

Vector construction and recombinant $N K G 2 F$ expression. The coding sequence of NKG2F was obtained from NCBI with the accession number of AF350018 and synthesized by Shanghai Sangon Biological Engineering Technology \& Services Co., Ltd. The NKG2F sequence was cloned into pET-28a (+) (Novagen, Madison, WI, USA) at the multiple cloning sites NdeI and XhoI. Constructed pET-28a (+)-NKG2F was transformed into E. coli Rosetta (DE3) (Novagen) strain. Transformed cells were grown in Luria Broth culture medium containing $34 \mu \mathrm{g} / \mathrm{ml}$ chloramphenicol and $30 \mu \mathrm{g} / \mathrm{ml}$ kanamycin. Expression of recombinant NKG2F was induced at exponential phase with $0.1 \mathrm{mM}$ isopropyl- $\beta$-d-thiogalactoside (IPTG). Cells were cultured for another $4 \mathrm{~h}$ at $37^{\circ} \mathrm{C}$, harvested by centrifugation at $12000 \mathrm{x}$ g for $10 \mathrm{~min}$ and resuspended in lysis buffer $(50 \mathrm{mM}$ Tris- $\mathrm{Cl}, 500 \mathrm{mM}$ $\mathrm{NaCl}, \mathrm{pH}$ 7.5). The cells were lysed with the French press and the supernatant and pellet were separated by centrifugation at $12000 \mathrm{x} \mathrm{g}$ for $20 \mathrm{~min}$. Pellet containing the target protein $\mathrm{NKG} 2 \mathrm{~F}$ was collected and thoroughly dispersed in urea wash buffer (2 M Urea, $500 \mathrm{mM} \mathrm{NaCl}, 20 \mathrm{mM}$ Tris, $\mathrm{pH}$ 8.89 ) or Triton wash buffer ( $2 \%$ Triton X-100, 2 M Urea, 500 $\mathrm{mM} \mathrm{NaCl}, 20 \mathrm{mM}$ Tris, $\mathrm{pH}$ 8.86) by sonication, followed with centrifugation at $6000 \mathrm{x}$ g for $10 \mathrm{~min}$, repeated 3 times. Pellet was analyzed by SDS-PAGE and stored at $-80^{\circ} \mathrm{C}$.

Mice. BALB/c mice were purchase from Shanghai Experimental Animal Center, Chinese Science Academy (Shanghai, China). All mice were maintained in a specific pathogenfree microenvironment, and received care in compliance with the guidelines outlined in the Guide for the Care and Use of Laboratory Animals. Mice used were between 8 and 10 weeks of age.

Cell lines. NKL cell lines (18), YT cell lines (19) were cultured in RPMI-1640 medium (Gibco, Grand Island, NY, USA) supplemented with $10 \%$ fetal bovine serum, $100 \mu \mathrm{g} / \mathrm{ml}$ streptomycin and $100 \mathrm{IU} / \mathrm{ml}$ penicillin (Gibco). NKL cell lines were maintained in culture with $100 \mathrm{IU} / \mathrm{ml} \mathrm{rhIL}-2$ (Changansheng, Chang Chun, China). All cells were cultured at $37^{\circ} \mathrm{C}$ in $5 \% \mathrm{CO}_{2}$ humidified atmosphere.
Western blotting. The protein supernatants were mixed in Laemmli loading buffer, boiled for $5 \mathrm{~min}$, and then loaded to SDS-PAGE. After electrophoresis, proteins were transferred onto nitrocellulose membranes (Millipore Corporation, Billerica, USA), and blotted against primary Abs overnight at $4{ }^{\circ} \mathrm{C}$. Membranes were washed with $0.1 \%$ (vol/vol) Tween20 in TBS (pH 7.6) and incubated with a 1:2500 dilution of horseradish peroxidase-conjugated secondary Abs for $60 \mathrm{~min}$ at room temperature. Protein bands were visualized by ECL reaction (Pierce, USA).

Mass spectrometry. Gel band of target protein was excised, destained twice with $0.2 \mathrm{ml}$ of $100 \mathrm{mM} \mathrm{NH} \mathrm{NCO}_{3} / 50 \%$ $\mathrm{ACN}$ for $45 \mathrm{~min}$, dehydrated for $5 \mathrm{~min}$ at room temperature in $200 \mu 1100 \%$ ACN and dried by Speed Vac. The dehydrated gel band was rehydrated with Trypsin Gold solution (Promega, WI, USA) at room temperature for $1 \mathrm{~h}$. More digestion buffer $\left(40 \mathrm{mM} \mathrm{NH} \mathrm{NCO}_{3} / 10 \% \mathrm{ACN}\right)$ was added to completely cover the gel band. Digestion was performed at $37^{\circ} \mathrm{C}$. The gel spots were incubated with $100 \mathrm{ml}$ of proteomics grade water for $10 \mathrm{~min}$ followed by $50 \mathrm{ml}$ of $50 \%$ ACN $/ 5 \%$ TFA for 60 min twice. All extracts were collected in a new microcentrifuge tube and dried by Speed Vac.

The tryptic peptides were resuspended in $0.1 \%$ formic acid analyzed by a Thermo LTQ linear IT MS (Thermo Electron, San Jose, CA) equipped with an Ettan ${ }^{\mathrm{TM}}$ MDLC HPLC system (General Electric Company, Piscataway, NJ). Peptides were on-line separated by a fused silica capillary column packed with C18 resin $(3.5 \mathrm{~mm}, 300 \AA$ A, Agilent Technologies, Santa Clara, CA) using a linear gradient of $0.1 \% \mathrm{v} / \mathrm{v}$ formic acid in water (solvent A) and ACN (solvent B) (4-60\% ACN over $50 \mathrm{~min}$ ). Mass spectra were acquired in a survey scan from 400 to $2000 \mathrm{amu}$ followed by five data dependent MS/MS scans (the most intense ion was selected and excluded for further selection for duration of $3 \mathrm{~min}$ ). MS/MS data were acquired using a 3-m/z unit ion isolation window and $30 \%$ relative collision energy. MS/MS data was analyzed by Bioworks version 3.2 software (Sequest, Thermo Electron) installed on a local server.

Preparation of polyclonal antibody. Polyclonal antibody against NKG2F was produced by repeatedly immunizing 


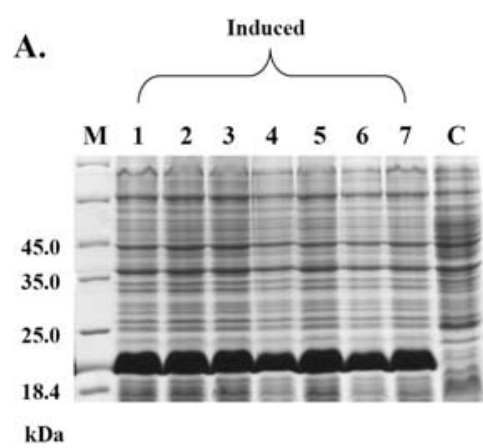

E. coli deposit

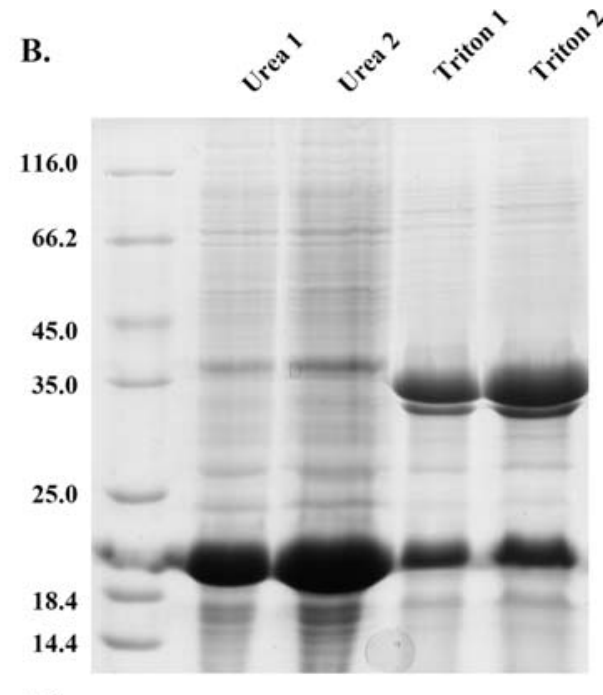

kDa

Figure 2. Purification of human NKG2F inclusion body. (A) SDS-PAGE analysis of the pellet of $E$. coli. Lane M was the marker. Lanes 1-7 were pellets of seven $E$. coli clones induced by $0.1 \mathrm{mM}$ IPTG. Lane C was the pellet of E. coli clone 7 without IPTG induction which was set as control. (B) Pellets of two clones were washed by urea buffer (lane urea 1 and urea 2) or Triton buffer (lane Triton 1 and Triton 2) in order to remove impurities.

8-10-week BALB/c mice (male) with NKG2F inclusion body. Purified NKG2F inclusion body was separated by SDS-PAGE and transferred onto nitrocellulose membrane. Specific bands of NKG2F were cut out and homogenized to prepare a homogenate mixture of inclusion body and nitrocellulose membrane in sterilized PBS. All the works were performed in sterilized condition. Homogenate mixture was i.p. injected into BALB/c mice once a week for 4 weeks. The mice serum was harvested as polyclonal antibody.

Isolation of peripheral blood mononuclear cells (PBMC). PBMCs (peripheral blood mononuclear cells) were isolated by Ficoll-Hypaque density gradient centrifugation (Beijing Solabrio Science \& Technology Co., Ltd., China) from heparinized venous blood samples obtained from healthy donors at Blood Center of Anhui Province, China as previously described $(20,21)$. PBMCs were maintained in RPMI-1640 medium (Gibco) supplemented with $10 \%(\mathrm{v} / \mathrm{v})$ fetal bovine serum (Yikesai, Shanghai, China) at $2 \times 10^{6} /$ well in a 12 -well cell culture plate, at $37^{\circ} \mathrm{C}$ in $5 \% \mathrm{CO}_{2}$.

Reverse transcription PCR. RNA was extracted from YT cell lines, NKL cell lines and PBMCs using TRIzol Reagent (Invitrogen, Carlsbad, CA, USA). RNA was reverse transcribed with M-MLV (Invitrogen). The primer sequences used in polymerase chain reaction (PCR) were as follows: NKG2F: sense, 5'-CCT GAA TAG AAG AAT GCA GAA AGC A-3'; anti-sense, 5'-AAA TAT TAT GAA GTC AGT TGA ATA CTA CAC AGA C-3' (15). ß-actin: sense, 5'-CGC GAG AAG ATG ACC CAG ATC-3'; anti-sense, 5'-TTG CTG ATC CAC ATC TGC TGG-3'. PCR was performed with $94^{\circ} \mathrm{C}$ for $30 \mathrm{sec}, 64^{\circ} \mathrm{C}$ for $1 \mathrm{~min}, 72^{\circ} \mathrm{C}$ for $1 \mathrm{~min}$, total 31 cycles and extended at $72^{\circ} \mathrm{C}$ for $10 \mathrm{~min}$.

Real-time PCR analysis. RNA was extracted from PBMCs stimulated by $1000 \mathrm{IU} / \mathrm{ml} \mathrm{IL-2}$ or $50 \mathrm{ng} / \mathrm{ml} \mathrm{IL-15,} \mathrm{respect-}$ ively for 24 or $48 \mathrm{~h}$. Cellular RNA $(1 \mu \mathrm{g})$ was reverse- transcribed with M-MLV (Invitrogen). The products (cDNA) were subjected to the real-time PCR analysis using a SYBRGreen Premix Ex Taq (Takara, Dalian, China) with the Rotor-Gene RG-3000A system (Corbett Research, Australia). Primers and PCR conditions were the same with Reverse Transcription PCR. Results were analyzed by Rotorgene 6 software (Corbett Research).

Flow cytometry analysis. PBMCs stimulated with cytokines (IL-2 or IL-15) were washed twice in washing buffer (PBS with 5\% FCS) and resuspended in 100- $\mu 1$ staining buffer (PBS with $0.5 \%$ BSA and $0.1 \% \mathrm{NaN}_{3}$ ) at $10 \% \mathrm{ml}$. Cells were blocked with mouse serum for $30 \mathrm{~min}$ at $4^{\circ} \mathrm{C}$, and stained with primary antibody (mice anti-NKG2F serum made by us) for $1 \mathrm{~h}$ at $4^{\circ} \mathrm{C}$. Cells were washed three times and stained with fluorochrome-conjugated secondary antibody (PE Rat anti-mouse) for $30 \mathrm{~min}$ at $4^{\circ} \mathrm{C}$, while isotype group was stained with PE Rat anti-mouse IgG. Cells were washed three times and stained with Alexa 488 mouse anti-human CD56, PerCPCY5.5 mouse anti-human CD3 for $30 \mathrm{~min}$ at $4^{\circ} \mathrm{C}$, while isotype group was stained with Alexa 488 mouse $\operatorname{IgG} 1$ and PerCP-CY5.5 mouse IgG1. Cells were washed three times and fixed with PBS containing $1 \%(\mathrm{w} / \mathrm{v})$ paraformaldehyde. Cells were acquired by FACSCalibur (BD, USA) and results were analyzed with WinMDI2.9 software.

\section{Results}

Recombinant expression of human NKG2F. NKG2F gene (NCBI Accession \#AF350018) was synthesized and inserted into vector pET-28a (+) between the restriction sites NdeI and XhoI containing a hexahistidine (6X His) tag and a thrombin digestion sequence to the $\mathrm{N}$-terminus of the recombinant protein NKG2F (Fig. 1A). The constructed vector pET-28a (+)-NKG2F was transformed into $E$. coli Rosetta (DE3) and induced with $0.1 \mathrm{mM}$ isopropyl- $\beta-d-$ thiogalactoside (IPTG) at exponential phase. IPTG induction 


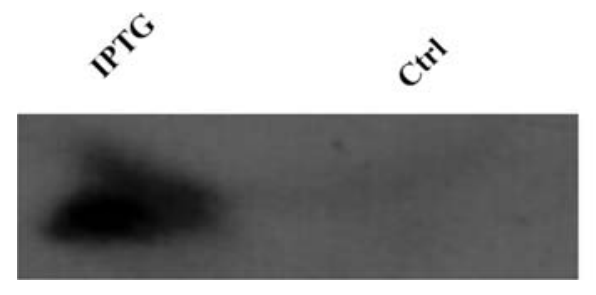

$\sim 20 \mathrm{kDa}$

Figure 3. Identification of NKG2F by anti-his Western blotting. Protein samples of IPTG induction E. coli (lane IPTG) and non-induction E. coli (lane Ctrl) were prepared for Western blotting. The anti-His antibody was used as the primary antibody.

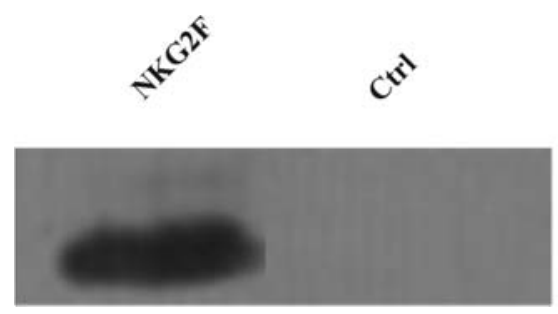

$\sim 20 \mathrm{kDa}$

Figure 4. Western blotting of human NKG2F by using polyclonal antibody. Recombinant NKG2F inclusion body was separated by SDS-PAGE. The target area on gel $(\sim 20 \mathrm{kDa})$ was transferred to $\mathrm{NC}$ membrane. Sera of NKG2F inclusion body immunized mice (NKG2F) and PBS injected mice (Ctrl) were used as primary antibody.

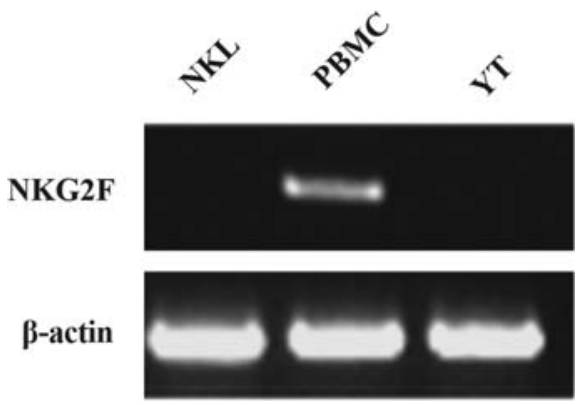

Figure 5. RT-PCR analysis of NKG2F expression. NKL cell lines, YT cell lines and PBMCs were analyzed for NKG2F transcripts by RT-PCR.

resulted in high expression of recombinant protein with a molecular weight of about $20 \mathrm{kDa}$ (Fig. 1B). SDS-PAGE analysis demon-strated that recombinant protein was only expressed in the pellet (Fig. 2A), none was detected in supernatant (data not shown). The pellet was further washed three times using either urea washing buffer or Triton washing buffer to improve the purity of the inclusion body. SDSPAGE results demon-strated that the inclusion body washed by urea washing buffer was of a higher purity with much less contaminated proteins (Fig. 2B).

To identify whether the $20-\mathrm{kDa}$ protein was recombinant NKG2F, anti-His (primary antibody) Western blotting was performed. IPTG induced $E$. coli showed a dark band at $20 \mathrm{kDa}$, while control (without IPTG induction) had no band, indicating that the $20-\mathrm{kDa}$ protein was recombinant NKG2F (Fig. 3). To further confirm the results of anti-his Western blotting, LC-MS/MS was used to sequence the target protein $(\sim 20 \mathrm{kDa})$. The results and the NKG2F sequence in database (UniProtKB/Swiss-Prot Accession \#O43908) matched well (Table I), indicating that the target protein was NKG2F.

Preparation of polyclonal antibody against human NKG2F. Polyclonal antibody was produced by immunizing 8-10-week $\mathrm{BALB} / \mathrm{c}$ mice (male) with the purified recombinant NKG2F protein as described in Materials and methods. To confirm the quality of the polyclonal antibody, we performed Western blotting. NKG2F inclusion body was separated by SDS-PAGE and transferred to nitrocellulose membrane. The polyclonal antibody was used as the primary antibody, and the serum of the control mice (PBS injection only) was used as control. Western blotting results demonstrated that the serum of NKG2F immunized mice was able to bind to NKG2F in contrast with the serum of control mice (Fig. 4), confirming the anti-NKG2F activity of the polyclonal antibody.

Up-regulated NKG2F expression of human NK cells after IL-2 or IL-15 stimulation. According to the results of RT-PCR, NKG2F was highly expressed in PBMCs, slightly expressed in NK92 cell lines, but not expressed in NKL cell lines or YT cell lines at mRNA level (Fig. 5). PBMCs were cultured in complete RPMI-1640 medium and stimulated with IL-2 or IL-15. NKG2F expression was evaluated after either 24 or $48 \mathrm{~h}$ by real-time PCR at mRNA level (Fig. 6) and by flow cytometry at protein level (Fig. 7). Both IL-2 and IL-15 stimulation up-regulated the expression of NKG2F of PBMCs at mRNA level (Fig. 6). As previous studies demonstrated that NKG2F was possibly expressed by NK cells (17), flow cytometry analysis was performed and primary NK cells

Table I. LC-MS/MS identification of recombinant protein.

\begin{tabular}{|c|c|c|c|c|c|c|c|}
\hline Protein name & MW (Da) & $\begin{array}{c}\text { NCBI } \\
\text { Accession no. }\end{array}$ & Peptide matched & $\mathrm{MH}^{+}$ & $\begin{array}{l}\text { Charge } \\
\text { state }\end{array}$ & Probability & Xcorr \\
\hline \multicolumn{8}{|l|}{ NKG2F } \\
\hline \multirow[t]{4}{*}{ (Homo sapiens) } & 18169.4 & 2980863 & KQRGTYSEVSLAQDPKR.Q & 1834.94060 & 2 & $2.28 \mathrm{E}-07$ & 2.54 \\
\hline & & & K.SSISGTKQEIFQVELNLQNASSDHQGNDK.T & 3174.52979 & 3 & $3.80 \mathrm{E}-14$ & 6.18 \\
\hline & & & R.GTYSEVSLAQDPK.R & 1394.67980 & 2 & $5.54 \mathrm{E}-11$ & 3.64 \\
\hline & & & R.GTYSEVSLAQDPKR.Q & 1550.78091 & 2 & $7.48 \mathrm{E}-14$ & 3.55 \\
\hline
\end{tabular}


A.

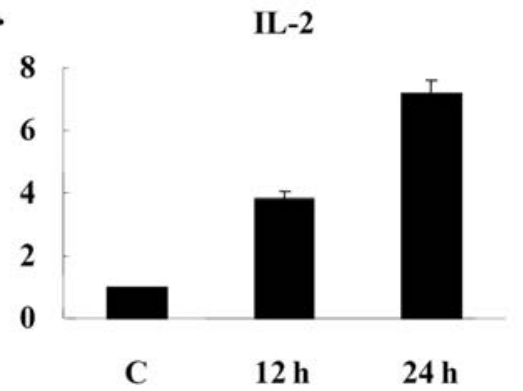

B.

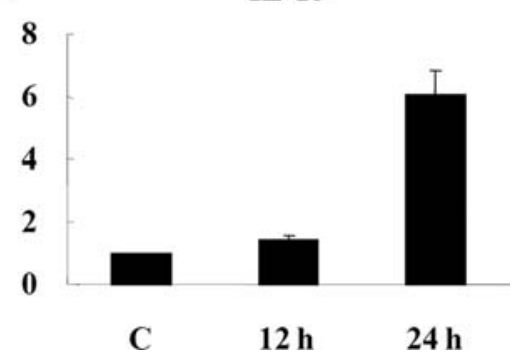

Figure 6. Real-time PCR analysis of NKG2F expression in human PBMCs after IL-2 or IL-15 stimulation. mRNA expression of NKG2F in PBMCs stimulated by $1000 \mathrm{IU} / \mathrm{ml} \mathrm{IL}-2$ or $50 \mathrm{ng} / \mathrm{ml} \mathrm{IL}-15$ respectively for 24 or $48 \mathrm{~h}$. Graphs show mean \pm SD, $\mathrm{n}=3$. C, PBMCs treated with PBS which was set as calibrator and assigned a relative concentration of 1 . AU, arbitrary unit.

A.

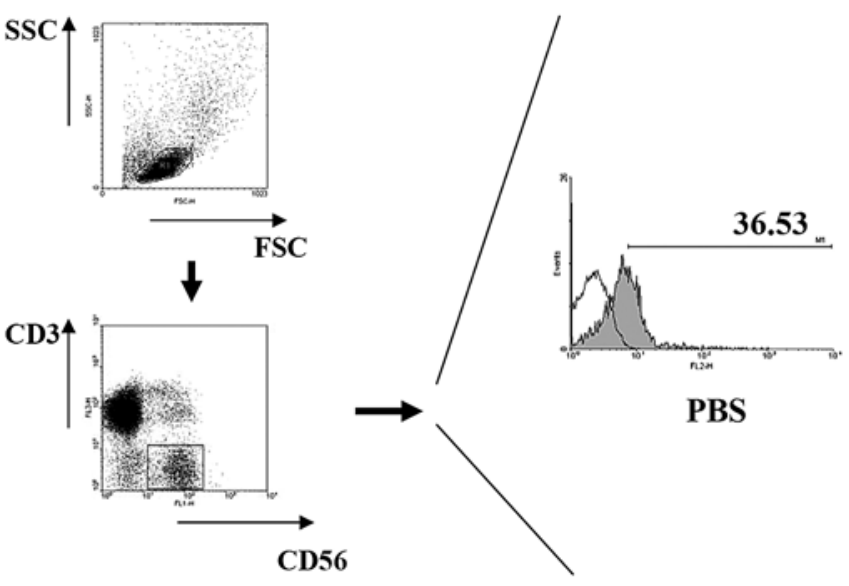

B.

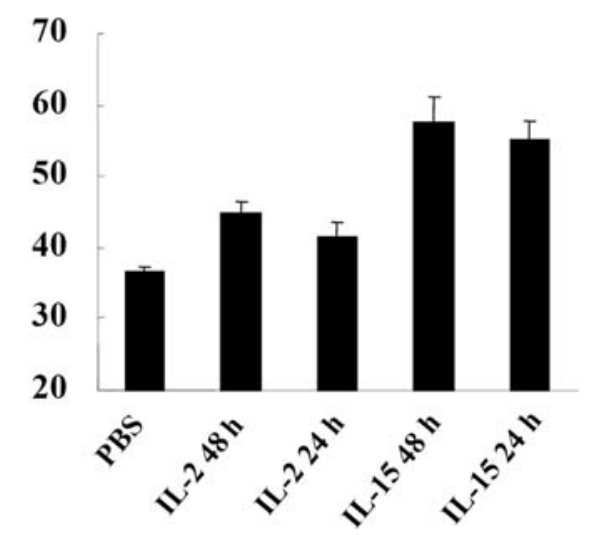

$\left(\mathrm{CD} 3 \mathrm{CD}^{-} 6^{+}\right)$were gated for analysis of NKG2F expression. It was revealed that $\mathrm{NKG} 2 \mathrm{~F}$ expression on primary NK cells was up-regulated by both IL-2 and IL-15 stimulation, and IL-15 was more efficient than IL-2 (Fig. 7).

\section{Discussion}

NKG2F gene encoding a putative protein which does not contain any lectin domain, was identified as a new member of NKG2 family in 1997 (16). Since lacking anti-NKG2F

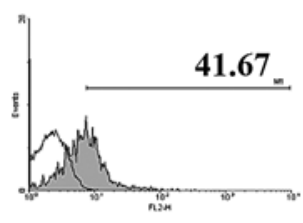

IL-2, 24 h

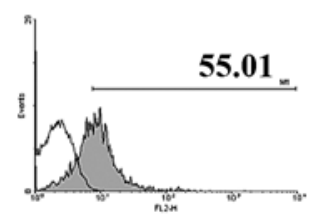

IL-15, 24 h

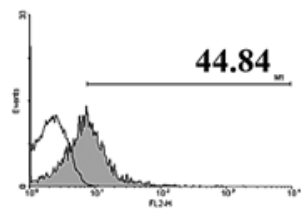

IL-2, 48 h

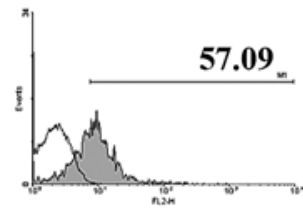

IL-15, 48 h
NKG2F

Figure 7. Flow cytometric analysis of surface NKG2F of human NK cells after IL-2 or IL-15 stimulation by using polyclonal antibody. (A) PBMCs were stimulated by $1000 \mathrm{IU} / \mathrm{ml} \mathrm{IL}-2$ and $50 \mathrm{ng} / \mathrm{ml} \mathrm{IL}-15$, respectively for 24 and $48 \mathrm{~h}$. Flow cytometry analysis was conducted. NK cells (CD3-CD56 ${ }^{+}$) were gated for NKG2F expression. PBS group was set as vehicle. Numbers indicated the percentage of NKG2F positive NK cells among total NK cells. (B) Percentages of NKG2F positive NK cells from three separate experiments were pooled. Mean + SEM shown.

antibody, previous studies have been mainly focusing on detecting the transcription rather than protein synthesis of $\mathrm{NKG} 2 \mathrm{~F}$ for a long time. NKG2F protein was found to be expressed in primary NK cells and NK cell lines by using an anti-NKG2F peptide sera specific for the C-terminus of $\mathrm{NKG} 2 \mathrm{C}$, -E and $-\mathrm{F}$ in Western blotting, in which NKG2F was found to associate with DAP12, an activating signal transducer, and thus NKG2F was speculated as an possible activating receptor (17). Due to the lack of recombinant NKG2F and antibody specifically against NKG2F, the functional study of NKG2F was hampered. In this study, we produced a polyclonal antibody against a full length but not a peptide of $\mathrm{NKG} 2 \mathrm{~F}$ for the first time, and found the antibody might be used not only to detect NKG2F protein in Western blotting as previously reported (17), but also firstly to examine the surface expression of NKG2F on NK cells in flow cytometry. 
Previous studies have demonstrated that IL-2 or IL-15 stimulation activated NK cells (22-25). According to the results of this study, either IL-2 or IL-15 stimulation resulted in up-regulation of NKG2F expression on NK cells. As NKG2F expression is consistent with NK cell activation, our results agree that NKG2F plays a positive role in NK cell activation. Moreover, it has been reported that Mycobacterium tuberculosis antigens can activate NK cells (26), indicating that microbe stimulation might regulate expression of NKG2F.

No ligands of $\mathrm{NKG} 2 \mathrm{~F}$ have been identified so far, that is why the progress in NKG2F functional study has been very slow. Similarly to this situation, NKG2D, another important activating receptor of NKG2 family, was first identified in human NK cells by Houchins and colleagues (27), but the biological functions of NKG2D were ignored until MHC class I chain related A/B $(\mathrm{MICA} / \mathrm{B})$ and UL16-binding proteins (ULBP) were identified as its ligands $(28,29)$. To further explore the biological functions of NKG2F, more work needs to be done to identify the ligands of NKG2F.

\section{Acknowledgements}

This study was supported by Natural Science Foundation of China (\#30801007, \#30721002, \#30870998, \#30630059) and Ministry of Science and Technology of China (973 Basic Science Project \#2007CB512405, \#2007CB512807, \#2006CB806504, \#2010CB911901).

\section{References}

1. Trinchieri G: Biology of natural killer cells. Adv Immunol 47: 187-376, 1989

2. Moretta A, Bottino C, Mingari MC, Biassoni R and Moretta L: What is a natural killer cell? Nat Immunol 3: 6-8, 2002.

3. Lanier LL: NK cell recognition. Annu Rev Immunol 23: 225-274, 2005

4. Farag SS and Caligiuri MA: Human natural killer cell development and biology. Blood Rev 20: 123-137, 2006.

5. Borrego F, Kabat J, Kim DK, et al: Structure and function of major histocompatibility complex (MHC) class I specific receptors expressed on human natural killer (NK) cells. Mol Immunol 38: 637-660, 2002.

6. Vales-Gomez M, Reyburn $\mathrm{H}$ and Strominger J: Interaction between the human NK receptors and their ligands. Crit Rev Immunol 20: 223-244, 2000.

7. Lanier LL: On guard-activating NK cell receptors. Nat Immunol 2: $23-27,2001$

8. Long EO and Rajagopalan S: HLA class I recognition by killer cell IG-like receptors. Semin Immunol 12: 101-108, 2000.

9. Carretero M, Palmieri G, Llano M, et al: Specific engagement of the CD94/NKG2-A killer inhibitory receptor by the HLA-E class IB molecule induces SHP-1 phosphatase recruitment to tyrosine-phosphorylated NKG2-A: evidence for receptor function in heterologous transfectants. Eur J Immunol 28: 1280-1291, 1998

10. Kabat J, Borrego F, Brooks A and Coligan JE: Role that each NKG2A immunoreceptor tyrosine-based inhibitory motif plays in mediating the human CD94/NKG2A inhibitory signal. J Immunol 169: 1948-1958, 2002.

11. Le Drean E, Vely F, Olcese L, et al: Inhibition of antigeninduced $\mathrm{T}$ cell response and antibody-induced NK cell cytotoxicity by NKG2A: association of NKG2A with SHP-1 and SHP-2 protein-tyrosine phosphatases. Eur J Immunol 28: 264 276,1998
12. Lanier LL, Corliss B, Wu J and Phillips JH: Association of DAP12 with activating CD94/NKG2C NK cell receptors. Immunity 8: 693-701, 1998.

13. Lanier LL, Corliss BC, Wu J, Leong C and Phillips JH: Immunoreceptor DAP12 bearing a tyrosine-based activation motif is involved in activating nk cells. Nature 391: 703-707, 1998.

14. Wu J, Cherwinski H, Spies T, Phillips JH and Lanier LL: DAP10 and DAP12 form distinct, but functionally cooperative, receptor complexes in natural killer cells. J Exp Med 192: 10591068,2000

15. Brostjan C, Bellon T, Sobanov Y, Lopez-Botet M and Hofer E: Differential expression of inhibitory and activating CD94/ NKG2 receptors on NK cell clones. J Immunol Methods 264: 109-119, 2002.

16. Plougastel B and Trowsdale J: Cloning of NKG2-F, a new member of the NKG2 family of human natural killer cell receptor genes. Eur J Immunol 27: 2835-2839, 1997.

17. Kim DK, Kabat J, Borrego F, Sanni TB, You CH and Coligan JE: Human NKG2F is expressed and can associate with DAP12. Mol Immunol 41: 53-62, 2004.

18. Robertson MJ, Cochran KJ, Cameron C, Le JM, Tantravahi R and Ritz J: Characterization of a cell line, NKL, derived from an aggressive human natural killer cell leukemia. Exp Hematol 24: 406-415, 1996.

19. Yodoi J, Teshigawara K, Nikaido T, et al: TCGF (IL2)-receptor inducing factor(s). I. Regulation of IL2 receptor on a natural killer-like cell line (YT cells). J Immunol 134: 1623-1630, 1985.

20. Boyum A: Isolation of mononuclear cells and granulocytes from human blood. Isolation of monuclear cells by one centrifugation, and of granulocytes by combining centrifugation and sedimentation at $1 \mathrm{G}$. Scand J Clin Lab Invest (Suppl) 97: 77-89, 1968.

21. Wang $\mathrm{Y}, \mathrm{Xu} \mathrm{H}$, Zheng $\mathrm{X}$, Wei H, Sun R and Tian Z: High expression of NKG2A/CD94 and low expression of granzyme B are associated with reduced cord blood NK cell activity. Cell Mol Immunol 4: 377-382, 2007.

22. Henney CS, Kuribayashi K, Kern DE and Gillis S: Interleukin-2 augments natural killer cell activity. Nature 291: 335-338, 1981.

23. Domzig W, Stadler BM and Herberman RB: Interleukin 2 dependence of human natural killer (NK) cell activity. J Immunol 130: 1970-1973, 1983.

24. Trinchieri G, Matsumoto-Kobayashi M, Clark SC, Seehra J, London L and Perussia B: Response of resting human peripheral blood natural killer cells to interleukin 2. J Exp Med 160: 1147-1169, 1984

25. Carson WE, Giri JG, Lindemann MJ, et al: Interleukin (IL) 15 is a novel cytokine that activates human natural killer cells via components of the IL-2 receptor. J Exp Med 180: 1395$1403,1994$.

26. Wang $\mathrm{C}$, Li J, Zheng $\mathrm{H}$, et al: The role of innate immune cells in the response of heat-treated mycobacterium tuberculosis (M.Tb) antigens stimulating PBMCS. Cell Mol Immunol 1: 467-470, 2004.

27. Houchins JP, Yabe T, McSherry C and Bach FH: DNA sequence analysis of NKG2, a family of related cDNA clones encoding type II integral membrane proteins on human natural killer cells. J Exp Med 173: 1017-1020, 1991.

28. Bauer S, Groh V, Wu J, et al: Activation of NK cells and T cells by NKG2D, a receptor for stress-inducible MICA. Science 285: 727-729, 1999.

29. Cosman D, Mullberg J, Sutherland CL, et al: ULBPs, novel MHC class I-related molecules, bind to CMV glycoprotein U116 and stimulate NK cytotoxicity through the NKG2D receptor. Immunity 14: 123-133, 2001. 